\title{
Wavelet and Curvelet Transformation based Image Fusion with ANFIS and SVM
}

\author{
Maninder Kaur \\ Department of CSE \\ CT Group of Institutions
}

\author{
Pooja \\ Assistant Professor, Department of CSE \\ CT Group of Institutions
}

\begin{abstract}
The Image fusion is a data fusion innovation which keeps images as main research substance which refers to the strategies that integrate multi-images of the same scene from multiple image sensor data or integrate multi images of the same scene at different times from single image sensor. In this paper we describes a novel image fusion method, is suitable for pan-sharpening of multispectral (MS) bands which are based on multi-resolution analysis. The low-resolution MS bands are sharpened by injecting high-pass directional details extracted from the high-resolution panchromatic (Pan) image by means of the Wavelet and Curvelet transform, which is a non-separable MRA, whose basis function are directional edges with progressively increasing resolution. We introduce a new method based on the Wavelet and Curvelet transform using Neural Network which represents edges better than wavelets in this paper. Therefore, edges play a fundamental role in image understanding and one important way to enhance spatial resolution is to enhance the edges. Wavelet and Curvelet-based image fusion method provides richer information in the spatial and spectral domains simultaneously
\end{abstract}

\section{General Terms}

Image Fusion, Image processing, pattern recognition

\section{Keywords}

Edge Detection, Wavelet and Curvelet Transform, NeuroFuzzy (ANFIS), Support Vector Machine (SVM)

\section{INTRODUCTION}

Multi-sensor Image fusion is the methodology of joining data from two or more images into a single image. Multi-sensor Image fusion is the process of combining more informative than any of the input images. Image fusion is a useful technique for merging similar sensor and multi-sensor images to enhance the information content present in the images.

Three different level fusion are:

- Feature level

- Pixel level

- Decision level.

These come under the fusion of data categorization. These Different algorithms of image fusion are used in different levels and have different and particular applications. Image fusion has several applications in various areas such as Medical Imaging, Remote sensing, Military applications, Robotics and so on [17]. In our previous paper [18] we have proposed a hybrid method for image fusion using ANFIS (Adaptive Neuro Fuzzy System) tool and SVM (Support Vector Machine) are show below in flow chart:

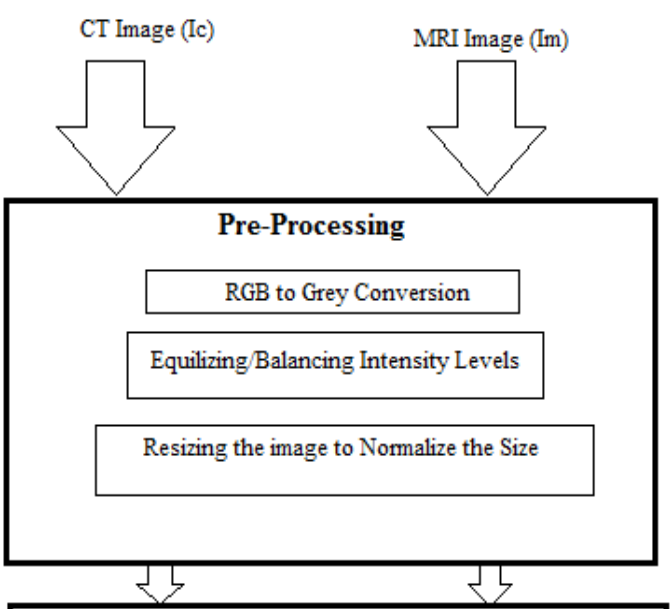

Applying Wavelet \& Curvelet Transform

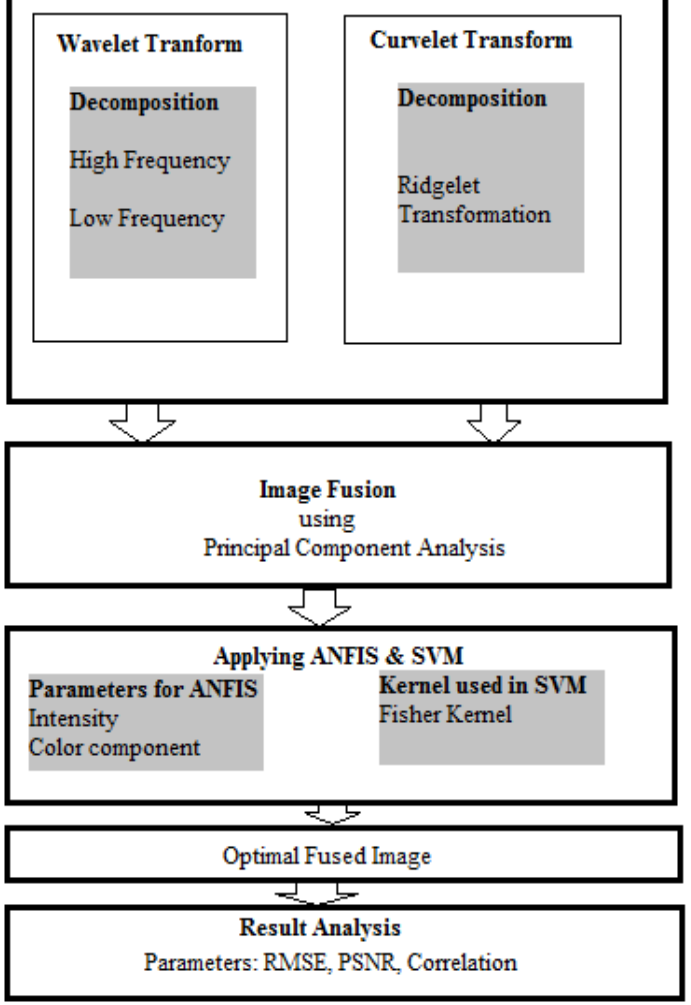

Figure 1 Image Fusion using Neuro-Fuzzy Algo \& SVM [18] 


\section{HYBRID METHOD (ANFIS-SVM)}

As shows in the above flow chart, our first step is to load images of CT and MRI.In our work,We have taken medical images. The database has been created for 30 images. We take $\mathrm{CT}$ and MRI images of same organs.

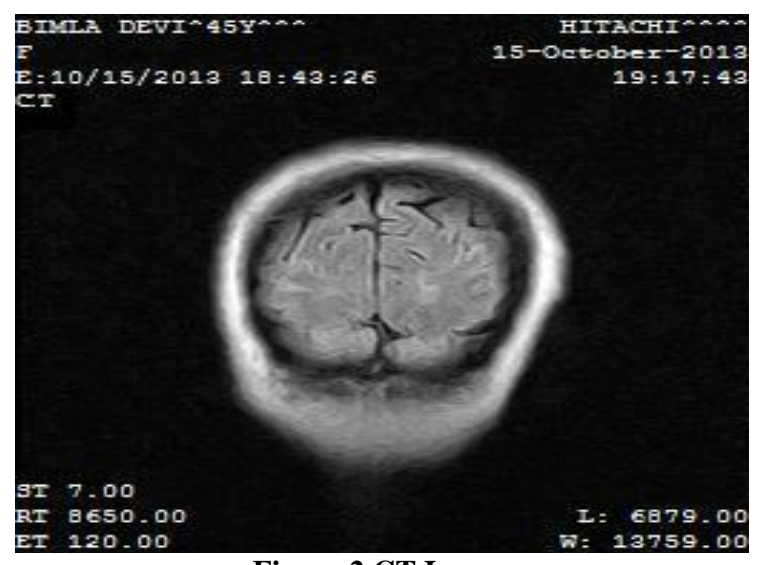

Figure 2 CT Image

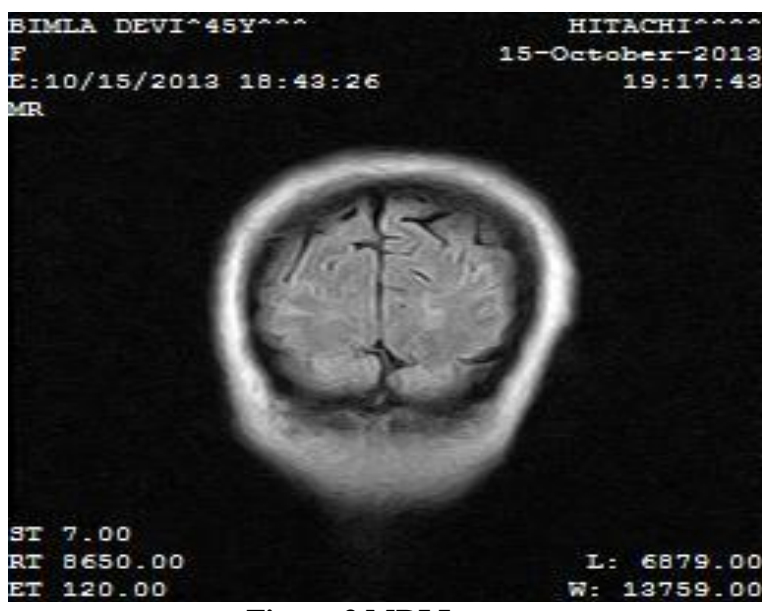

Figure 3 MRI Image

\section{PHASES OF PROPOSED WORK}

\subsection{Pre processing}

Two images taken in different angles of scene sometimes cause distortion. The objects are the same but the shapes change a little. At the starting of fusing images, make sure that each pixel of images at correlated images has the connection between images in order to fix the problem of distortion; image registration can do this [20]. Let us consider the preprocessing of CT/MRI images:

1. Firstly, convert the both images R,G,B components into grey color.

2. Equating and balancing intensity of grey color image.

3. Resize the image to normalize size.

\subsection{Multiwavelet Image Fusion Algorithm:}

1. First, two source input images (CT and MRI) are registered initially.

2. Decomposition steps are performed for both images.

3. Maximum frequency fusion rule (PCA) used for the fusion of the wavelet coefficients.
4. Apply ANFIS and SVM on the fused image.

5. Inverse multiwavelet transform performed to obtain the fused images.
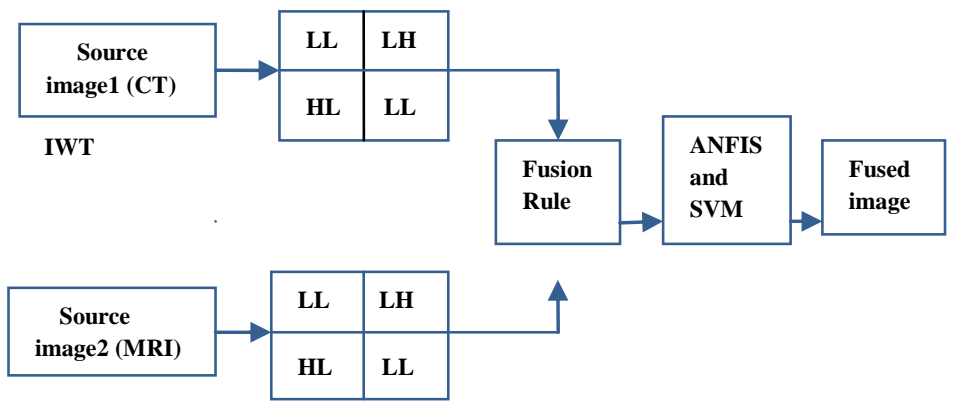

Wavelet Coefficients

Figure.4 Block diagram of wavelet transformation for image fusion

The following diagram shows the decomposition of CT and MRI images. Multiwavelet transformation applied on both images.

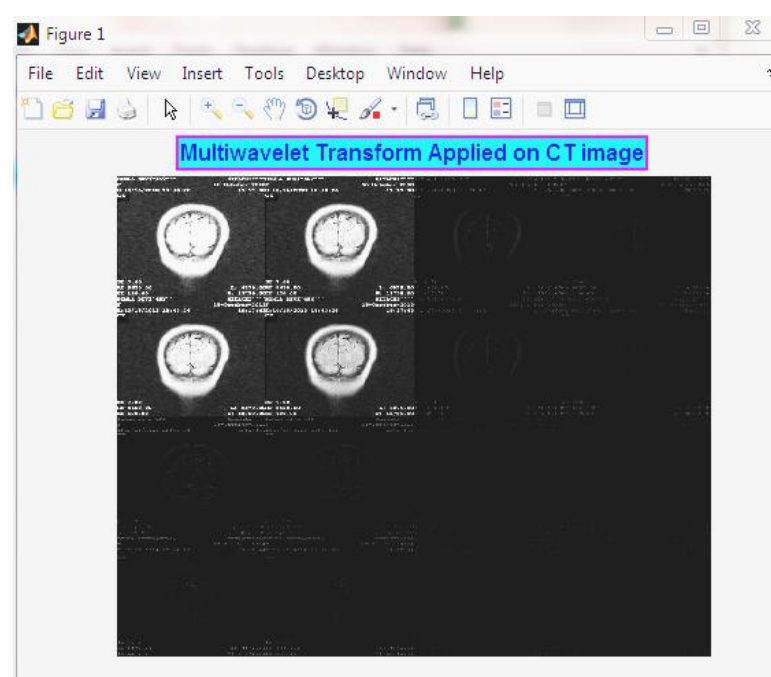

Figure.5 Decomposition of CT image with multiwavelet transformation

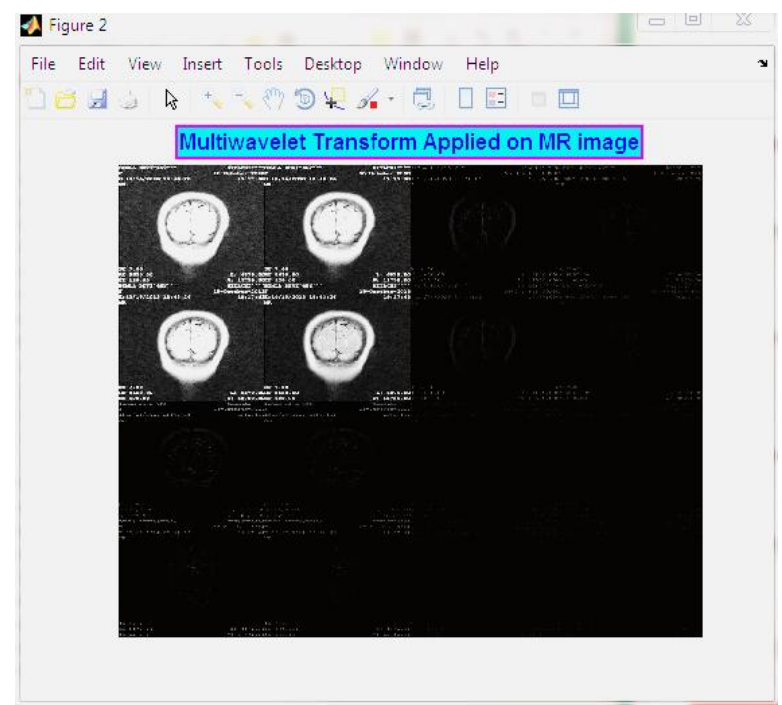

Figure.6 Decomposition of MRI image with multiwavelet transformation 


\subsection{Curvelet Image Fusion Algorithm:}

The algorithm for fusing images used for Curvelet transform is explained as follows [17]:

1. The two input images are initially registered.

2. Each input image is then analyzed and a set of curvelet (Ridgelet) coefficients are generated.

3. The maximum frequency rule (PCA) used for the fusion of the Curvelet or Ridgelet coefficients.

4. Apply ANFIS and SVM on the fused image.

5. Finally the Inverse Curvelet transform step is performed to obtain the fused images.

CVT

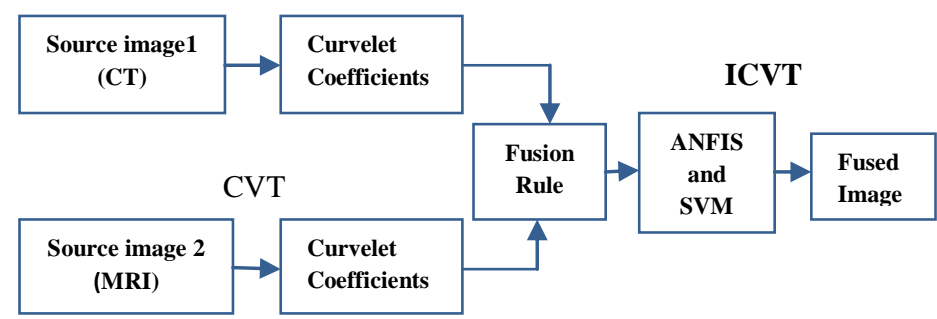

Figure.7 Block diagram of curvelet transformation for image fusion

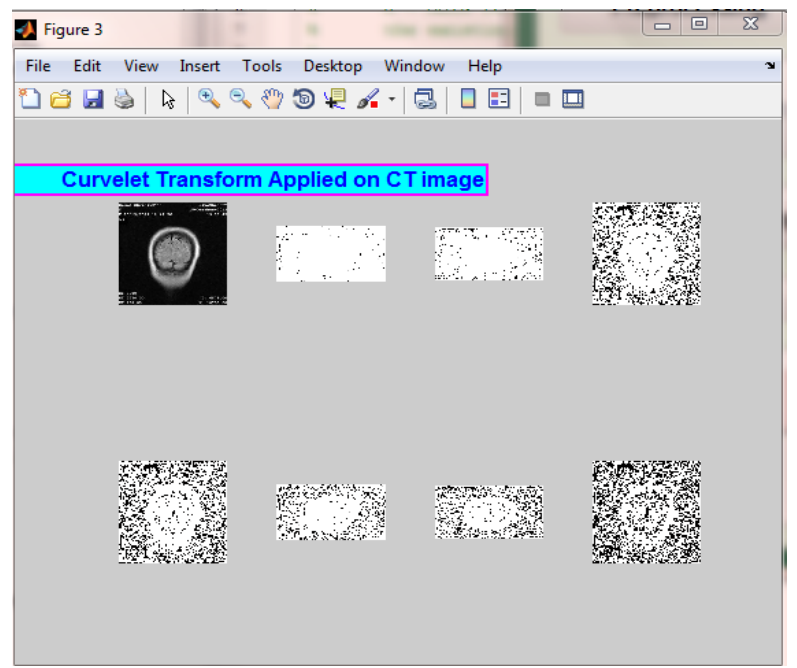

Figure.8 Curvelet transformation applied on CT image

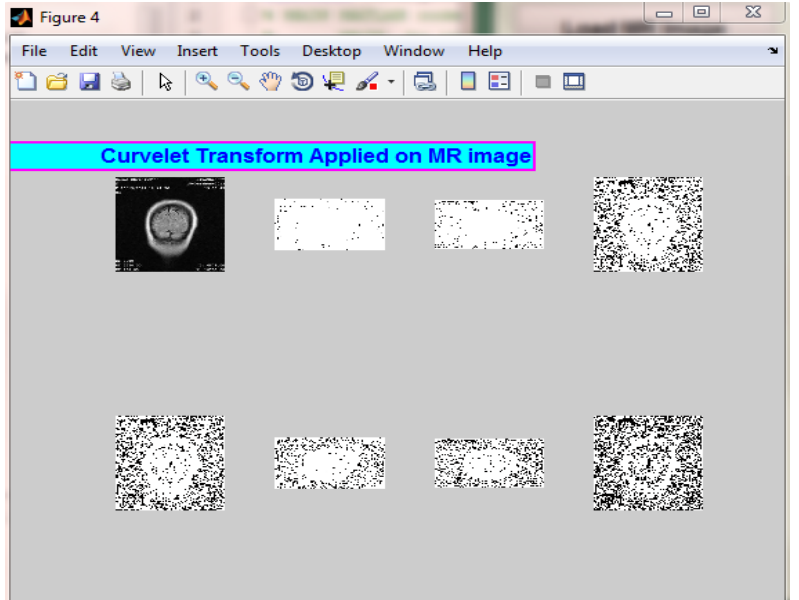

Figure.9 Curvelet transformation applied on MRI image

The figure 8 \& 9 shown above are displaying the decomposition of both (CT and MRI) images. Curvelet transformation applied on both source images.

\subsection{Image Fusion Method}

3.4.1 Principal Component Analysis (PCA): PCA is a vector space transform used for reducing the multidimensional data sets to lower dimensions [17]. In PCA the following steps are used:

- Firstly, column vectors are generated from the input images and then calculate the covariance matrix of two column vectors.

- The two terms, Eigen values and Eigen vectors are calculated of the covariance matrix.

- Normalize the column vector and the values of the normalized Eigen vector act as the weight values which are multiplied with each pixel of the input images.[17]

3.5 Adaptive Neuro Fuzzy Inference System This ANFIS methodology comprises of a hybrid system of fuzzy logic and neural network technique [18]. As show in the below (figure.10), in this hybrid method, First an initial Fuzzy model with its input variables, we load data from the workspace and then train and test the input image. After that, Generate a FIS, with the help of rules extracted from the input output data of the system. Next step is to train the FIS, we have using hybrid optimization method with two parameters Intensity and color component of an image. At last to produce the final input, test the final output of FIS

The structure of ANFIS is shown in (figure 11) the right most nodes show the input. Three membership functions are used for each input. The blue circles represent the usage of and rule. Output represented by the right most nodes. By Using ANFIS algorithm, the fused coefficients are obtained. The fused image is obtained by applying inverse transform to the fused coefficients. 


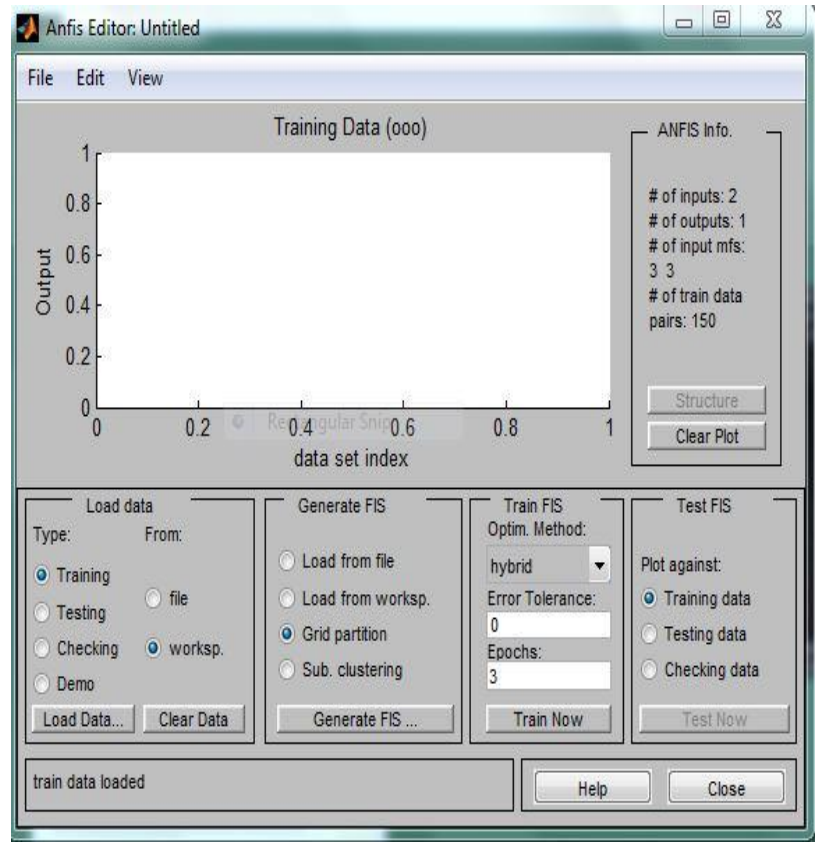

Figure.10 ANFIS Editor Tool

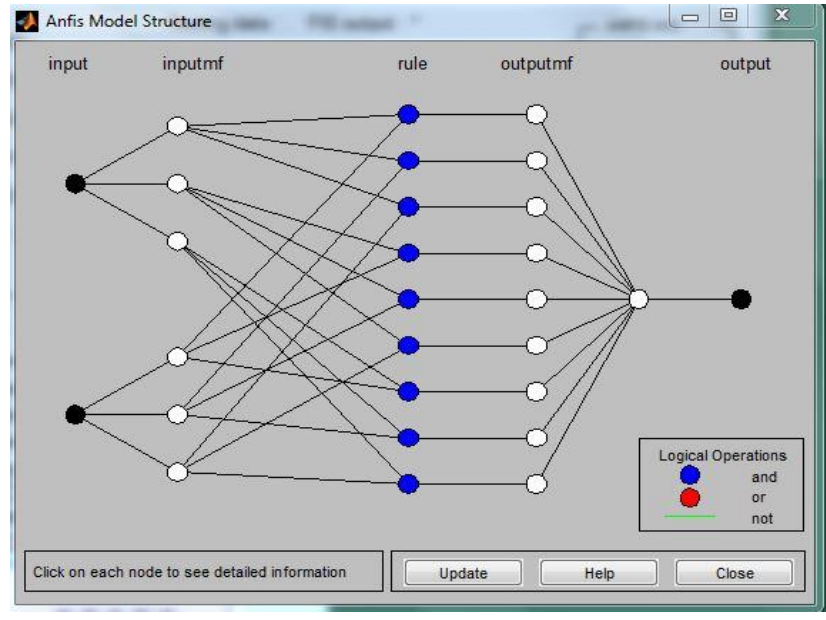

Figure.11 ANFIS structure for proposed algorithm

\subsection{Support Vector Machine (SVM):}

A SVM is a classification method that discriminates between two classes, by fitting an Optimal Separating Hyper plane (OSH) (Gunn, 1998). It actually maximizes the margin between two classes of training data [20].

We used Fisher kernel in this work. The following is SVM algorithm which we have used in our work.

\section{Algorithm 1 Simple SVM}

Candidate SV-\{closest pair from opposite classes\}

While there are violating points do

Find a violator

Candidate $\mathrm{SV}=$ candidate $\mathrm{SV} \mathrm{U}$ violator

If any $\alpha \rho<0$ due to addition of $\mathrm{c}$ to $\mathrm{S}$ then

candidate $\mathrm{SV}=$ candidate $\mathrm{SV} / \mathrm{p}$

On repeat till all such points are pruned

End if

End while

\subsection{Performance Measures}

\subsubsection{Peak Signal to Noise Ratio (PSNR)}

The PSNR block computes the peak signal-to-noise ratio, in decibels, between two images. It represents a measure of the peak error. The PSNR is the ratio between the maximum possible power of a signal and the power of corrupting noise that affects the fidelity of its representation [19].

The equation of PSNR of the fusion result is defined as follows:

PSNR $=10 * \log \left[L^{2} /\right.$ RMSE $]$

\subsubsection{Root Mean Square Error (RMSE)}

RMSE is used reference based metric is and it is calculated by using the following equation:

$\mathbf{R M S E}=1 / \mathrm{MN} \sum_{m=1}^{M} \sum_{\mathrm{n}=1}^{\mathrm{N}}(\mathrm{R}(\mathrm{m}, \mathrm{n}) \mathrm{F}(\mathrm{m}, \mathrm{n}))$

The performance of the fusion algorithm is better, if the RMSE value is smaller.

\subsection{3 $\operatorname{ENTROPY~}(H)$}

The entropy measure of information content of an image. If after fusing the value of entropy becomes elevated, it indicates that the information enhances and the fusion performances are better.

$$
\mathbf{E}=\sum_{g=0}^{L-1} p(g) \log 2 p(g)
$$

\subsubsection{Correlation Coefficient (CC)}

The correlation coefficient is the measure the closeness or similarity in small size structures between the original and the fused images. It can vary between -1 and +1 values. The Values closer to +1 shows that the reference and fused images are highly similar while the values closer to -1 indicate that the images are highly dissimilar.

$$
\mathrm{CC}=2 \mathrm{CrfCr}+\mathrm{Cf}
$$

Where

$$
\begin{aligned}
\mathrm{Cr} & =\mathrm{Ir}(\mathrm{I}, \mathrm{j}) \mathrm{NJ}=1 \mathrm{Mi}=1 \\
\mathrm{Cf} & =\text { If } \mathrm{NJ}=1 \mathrm{Mi}=1(\mathrm{I}, \mathrm{j}) \\
\mathrm{Crf} & =\text { If } \mathrm{Nj}=1 \mathrm{Mi}=1 \mathrm{I}, \mathrm{j} \text { If(i,j) }
\end{aligned}
$$

\subsubsection{Bit Error Ratio (BER)}

Bit error rate, BER is a key parameter that is used in assessing systems that transmit digital data from one location to another. As the name implies, a bit error rate is defined as the rate at which errors occur in a transmission system. It can be directly translated into the number of errors that occur in a string of a stated number of bits. The Bit error ratio defines following simple formula:

$$
\text { BER }=\text { No of Errors/Total no of bits sent }
$$

\section{THE EXPERIMENTAL RESULTS}

For image fusion, we take 28 Computed Tomography (CT) and 28 Magnetic Resonance (MR) images of Bimla Devi (45y) of different organs from medical scanning centre.MR and CT images are taken as the source images. CT image shows the bone information like bone injuries, lung and chest imaging and the MR image shows the soft tissues information. Multiwavelet and curvelet transform are applied on the source images and then PCA (Principal Component Analysis) fusion method applied on transform coefficients. In the following figures, result of proposed algorithm is shows. The following figure shows the graphical representation of Correlation Coefficient between the previous and proposed work and it is 
clear from the figure that the $\mathrm{CC}$ is better in our algorithm than the previous work.

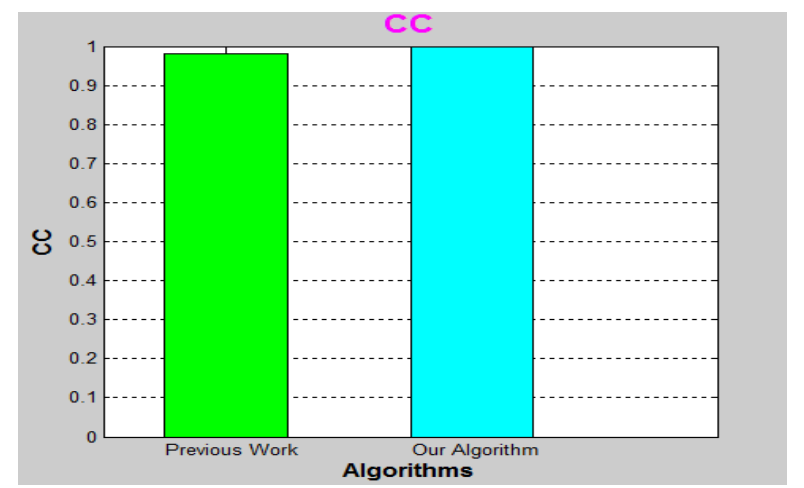

Figure 12: Correlation Coefficient of previous and proposed work

The following table shows the comparison of values of Correlation Coefficient (CC) between the previous and proposed method. This shows the better Correlation Coefficient (CC) values than the previous method.

Table 1: Comparison of Correlation Coefficient between previous and proposed work

\begin{tabular}{|c|c|c|r|r|r|}
\hline \multirow{2}{*}{ CC } & \multicolumn{5}{|c|}{ Image Number } \\
\cline { 2 - 6 } & 1 & 2 & 3 & 4 & 5 \\
\hline $\begin{array}{c}\text { Proposed } \\
\text { Work }\end{array}$ & 0.9998 & 0.9737 & 0.9275 & 1.2111 & 0.9702 \\
\hline $\begin{array}{c}\text { PCA } \\
\text { method }\end{array}$ & 0.9818 & 0.9639 & 0.9200 & 1.2033 & 0.9592 \\
\hline
\end{tabular}

The following figure shows the graphical representation of Bit Error Ratio (BER) between the previous and proposed work.

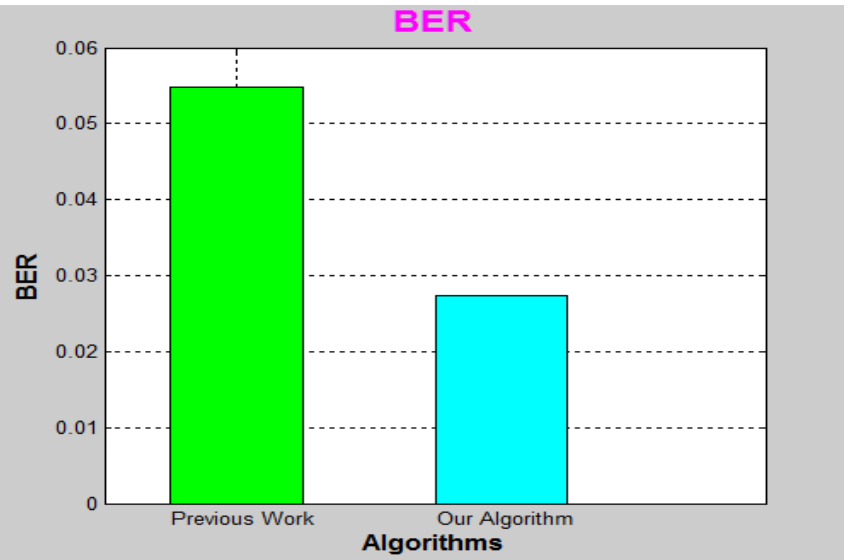

Figure 13: Bit Error Rate of previous and proposed work

The following table shows the comparison of values of Bit Error Ratio (BER) between the previous and proposed method. This shows the better Bit Error Ratio (BER) values than the previous method.

Table 2: Comparison of BER between previous and proposed work

\begin{tabular}{|l|c|c|c|c|c|}
\hline \multirow{2}{*}{ BER } & \multicolumn{5}{|c|}{ Image number } \\
\cline { 2 - 6 } & 1 & 2 & 3 & 4 & 5 \\
\hline $\begin{array}{l}\text { Proposed } \\
\text { Work }\end{array}$ & 0.0274 & 0.3288 & 0.2202 & 0.3512 & 0.2832 \\
\hline $\begin{array}{l}\text { PCA } \\
\text { method }\end{array}$ & 0.0547 & 0.6576 & 0.4404 & 0.7023 & 0.5664 \\
\hline
\end{tabular}

The following figure, which shows the graphical representation of Entropy $(\mathrm{H})$ between the previous and proposed work.

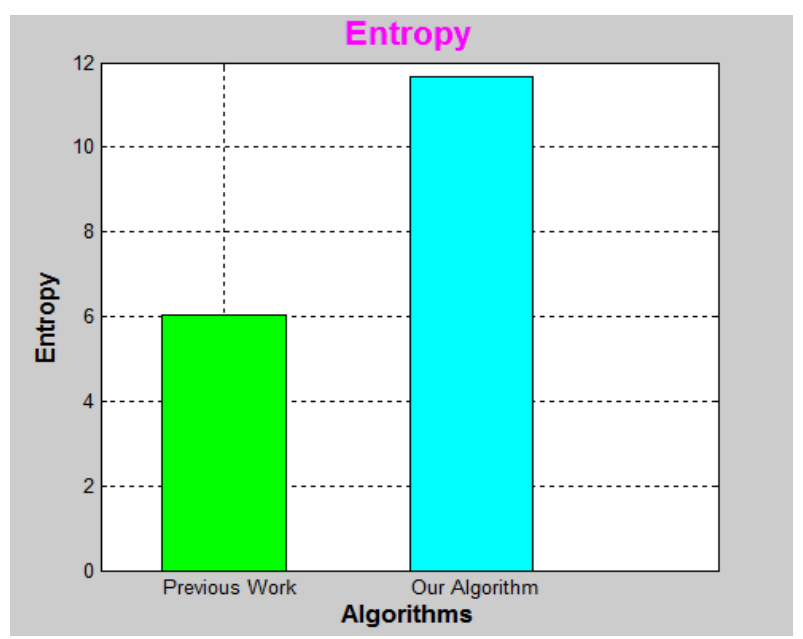

Figure 14: Entropy of previous and proposed work

The Table 3 shows the comparison of values of Entropy $(\mathrm{H})$ between the previous and proposed method. This shows the better Entropy values than the previous method.

Table 3: Comparison of Entropy between previous and proposed work

\begin{tabular}{|c|c|c|c|c|c|}
\hline \multirow{2}{*}{ Entropy } & \multicolumn{5}{|c|}{ Image Number } \\
\cline { 2 - 6 } & 1 & 2 & 3 & 4 & 5 \\
\hline $\begin{array}{c}\text { Proposed } \\
\text { Work }\end{array}$ & 11.6790 & 12.6850 & 9.6216 & 11.1858 & 11.3970 \\
\hline $\begin{array}{c}\text { PCA } \\
\text { method }\end{array}$ & 6.0453 & 6.0453 & 6.6026 & 6.3947 & 6.6957 \\
\hline
\end{tabular}

The figure which shows below, representation the graphical structure of Peak Signal to Noise Ratio (PSNR) between the previous and proposed work.

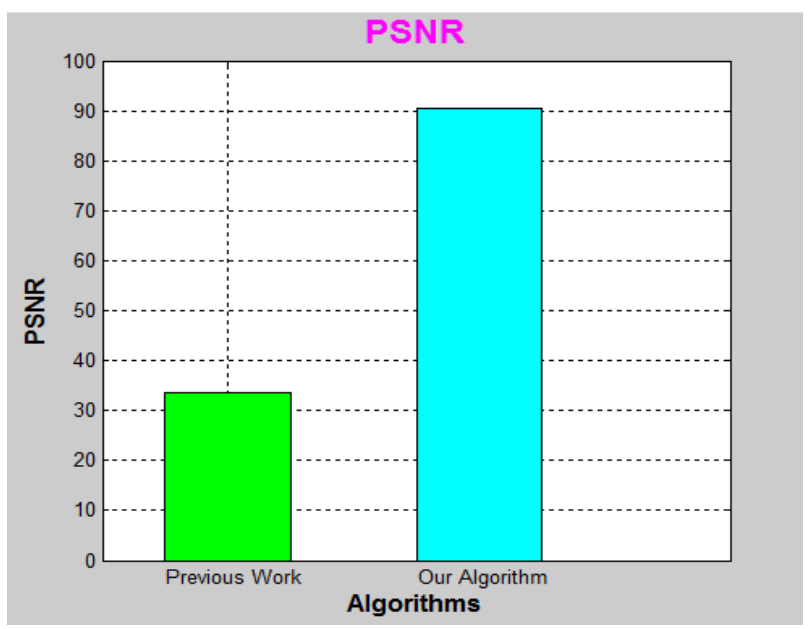

Figure15: PSNR of previous and proposed work

The following table (Table 4) shows the comparison of values of Peak Signal to Noise Ratio (PSNR) between the previous and proposed method. This shows the better Peak Signal to Noise Ratio (PSNR) values than the previous method. 
Table 4: Comparison of PSNR previous and proposed work

\begin{tabular}{|c|c|c|c|c|c|}
\hline \multirow{2}{*}{ PSNR } & \multicolumn{5}{|c|}{ Image Number } \\
\cline { 2 - 6 } & 1 & 2 & 3 & 4 & 5 \\
\hline $\begin{array}{c}\text { Proposed } \\
\text { Work }\end{array}$ & 90.4196 & 50.8524 & 53.0665 & 54.0090 & 53.0925 \\
\hline $\begin{array}{c}\text { PCA } \\
\text { method }\end{array}$ & 33.5805 & 33.5805 & 33.8095 & 33.6326 & 33.7651 \\
\hline
\end{tabular}

The following figure, which shows the graphical representation of Root Mean Square Error (RMSE) between the previous and proposed work

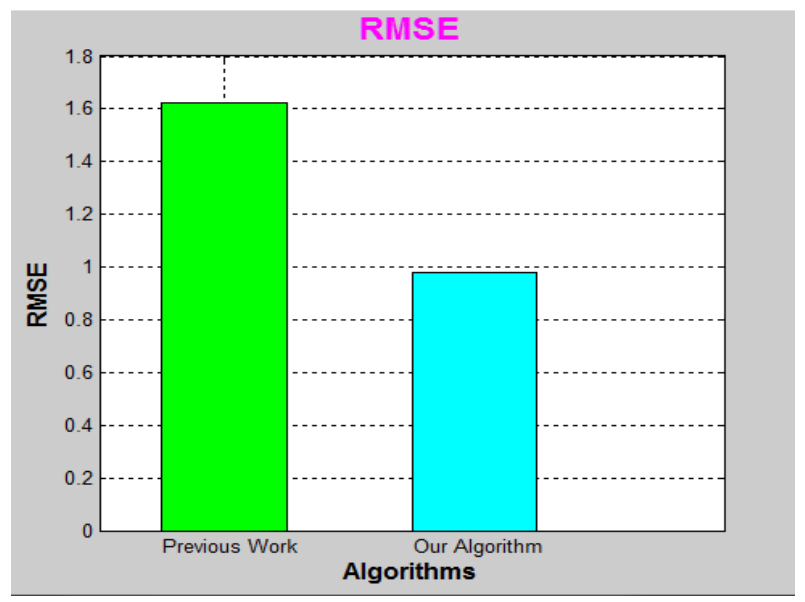

Figure 16: RMSE of previous and proposed work

The following table shows the comparison of values of Rot Mean Square Error (RMSE) between the previous and proposed method. This shows the better Root Mean Square Error (RMSE) values than the previous method.

Table 5: Comparison of RMSE between previous and proposed work

\begin{tabular}{|l|c|c|c|c|c|}
\hline \multirow{2}{*}{ RMSE } & \multicolumn{5}{|c|}{ Image Number } \\
\cline { 2 - 6 } & 1 & 2 & 3 & 4 & 5 \\
\hline $\begin{array}{l}\text { Proposed } \\
\text { Work }\end{array}$ & 0.9796 & 0.7282 & 0.5643 & 0.5063 & 0.5626 \\
\hline $\begin{array}{l}\text { PCA } \\
\text { method }\end{array}$ & 1.6226 & 1.6226 & 1.0025 & 1.9205 & 1.3309 \\
\hline
\end{tabular}

\section{CONCLUSION}

The paper has introduced another pattern in the combination of digital image, MRI and CT images which are taking into account the Curvelet and Wavelet transform. A comparison study has been made between the traditional wavelet fusion algorithm and the proposed Curvelet fusion algorithm. The experimental study demonstrates that the application of the Curvelet transform in the fusion of MR and CT images is better than the use of the traditional wavelet transform. The acquired Curvelet fusion results have minimum MSE and maximum PSNR than in wavelet fusion transform. At last, these fusion methods are used to detect brain tumors. In vision, the Neuro-fuzzy (ANFIS) is proposed for image fusion in this paper acquires better fusion result. Our work shows, Curvelet fusion characteristic are superior to wavelet transform.

\section{REFERENCES}

[1] Smt.G. Mamatha (Phd), L.Gayatri 2012."An image fusion using wavelet and curvelet transforms"'.Global Journal of Advanced Engineering Technologies, Volume 1 , Issue-2.

[2] T. Ranchin and L. Wald 2000, "Fusion of High Spatial and Spectral Resolution images: The ARSIS Concept and Its Implementation," Photogrammetric Engineering and Remote Sensing, volume. 66, pp. 49-61.

[3] L. Wald, T. Ranchin and M. Mangolini 1997, "Fusion of Satellite images of different spatial resolution: Assessing the quality of resulting images," Photogrammetric Engineering and Remote Sensing, volume. 63, pp. 691699.

[4] J. Nunez, X. Otazu, O. Fors, A. Prades, V. Pala and R. Arbiol 1999, "Multiresolution-based image fusion with additive wavelet decomposion," IEEE Transactions on Geoscience and Remote sensing, volume. 37, pp. 12041211.

[5] J.Cand'es1999, "Harmonic analysis of neural networks," Application Computer Harmonic. Analysis,volume. 6, pp. 197-218.

[6] J. L. Starck, E. J. Cand es and D. L. Donoho 2002, "The curvelet transform for image denosing," IEEE Trans. Image Processing, volume. 11, pp. 670-684.

[7] J. L. Starck, E, J. Cand'es, and D. L. Donoho 2003, "Gray and Color Image Contrast Enhancement by the Curvelet Transform," IEEE Trans. Image Processing, volume. 12 , pp. 706-717.

[8] E.J.Candes 1998, "Ridge lets: Theory and Applications," Ph.D. Thesis, Department of Statistics, Stanford University, Standford, CA.

[9] D. L. Donoho 1998, "A theory for multi-resolution signal decomposition: the wavelet representation," IEEE Pattern Anal. and Machine Intell., volume. 11, no. 7, pp. 674693".

[10] D. L. Donoho 2003, "Orthonormal ridge lets and linear singularities," SIAM J. Math Analysis, volume. 31, pp. 1062-1099.

[11] M. I. Smith, J. P. Heather 2005, "Fusion Technology Review of Image in 2005," Proceedings of the SPIE, Volume 5782, pp. 29-45.

[12] Yong Yang 2010, "Multi modal Medical Image Fusion through a New DWT Based Technique", 4th International Conference on Bioinformatics and Biomedical Engineering, pp 1-4.

[13] Chandrakanth.R, SaibabaJ, Varadan.G, Raj.PA 2011,"Fusion of High Resolution Satellite SAR and Optical Images "International Workshop on MultiPlatform/Multi-Sensor Remote Sensing and Mapping, pp 1-6.

[14] T.S.Anand, K.Narasimhan, P.Saravanan 2012 'Performance Evaluation of Image Fusion Using the Multi-Wavelet and Curvelet Transforms" IEEE, International Conference On Advances In Engineering, Science And Management. 
[15] Ms.Maninder kaur, Ms.Pooja 2015, 'Optimal Image Fusion using Neuro-Fuzzy Algorithm and SVM" Australian Journal of Information Technology and Communication Volume II,ISSN 2203-2843

[16] R.J.Sapkal, S.M.Kulkarni 2012,'Image Fusion based on Wavelet Transform for Medical Application' International Journal of Engineering Research and Applications (IJERA) ISSN: 2248-9622

[17] D. Egfin Nirmala, A. Bibin Sam Paul and V. Vaidehi 2013,' improving independent component analysis using support vector machines for multimodal image fusion" Journal of Computer Science 9 : 1117-1132.

[18] Navneet kaur, Madhu Bahl 2014 "Review on: Image Fusion using Wavelet and Curvelet Transform"International Journal of Computer Science and Information Technologies,Volume.5.

[19] Johnson Suthakar, J.Monica Esther M.E, D.Annapoorani, F.Richard Singh Samuel 2014“ Study of Image Fusion-Techniques, Method and Applications"International Journal of Computer Science and Mobile Computing, Volume. 3. 